\title{
Data Structures in Robot Navigation Optimized by Adaptive Straightness
}

\author{
Leoncio Claro Barros Neto \\ Escola Politécnica \\ Universidade de São Paulo (USP) \\ São Paulo, Brasil
}

\author{
André Riyuiti Hirakawa \\ Escola Politécnica \\ Universidade de São Paulo (USP) \\ São Paulo, Brasil
}

\begin{abstract}
Utilizing adaptive finite automaton (AFA) such as motion automaton, we propose an alternative for the available researches on data structures in robotics navigation, in which trajectories are made up of straight line segments. Software is modeled by a set of rules as systems of state machines to cover the complete space environment of the robot. The formalism of adaptive digitized straight line segments (ADSLS) is applied for data representation, aiming to exploit its ability to express tolerances, scalability, errors and deviations in angle or in length of segments. Consequently, ADSLS is shown by simulations to be effective to represent the complexities of real world scenarios of a robot; furthermore, it is able to adapt, reacting to circumstance stimuli in a single pass, also presenting learning capability.
\end{abstract}

\section{General Terms:}

Applications of Computer Science in Modeling, Reconfigurable Computing

\section{Keywords:}

Digital Geometry, Robotics, Pattern Recognition, Automata, Error Recovery

\section{INTRODUCTION}

Classically, robotic agents are usually equipped with sensor systems and actuators for perception and action, respectively, in the physical space to which they are integrated. In mobile robotics, which yielded notable researches since the 1970s, besides sensors and actuators, robots also are equipped with systems that allow them to move through the environment in order to perform activities of greater complexity than traditional robotics.

Navigation is understood as a process or activity to plan and direct a trajectory, a route or a path for a mobile robot. It is an activity that an autonomous robot performs with relative precision according to the task in order to move safely from one location to another location without getting lost or colliding with other objects [23].

Regarding the precision requirement in trajectory planning, [5] says that Cartesian space trajectory planning is commonly used because it is a straightforward and relatively simple concept. The cost of this simplification is the transformation from Cartesian to joint coordinates in real-time, causing the highly computational complexities involved in trajectory planning and coordinate transformation.

[5] also points out that Cartesian paths have been approximated, as an alternative, by joint trajectories; for example, intermedi- ate knot points are selected on a Cartesian line segment in Euclidean space which are further interpolated by joint trajectories, commonly cubic and quartic spline functions. Accordingly, the translation and rotation deviation errors between a Cartesian path and the corresponding interpolated approximate joint trajectories can be adjusted to satisfy specified tolerances so that approximation errors are reduced by using some criterion. Hence the actual implementation of on-line Cartesian-based path planning algorithms have been hindered by the computational complexities involved [5], also taking into account that modern mobile robots are now applied in various domains with spurious interferences and uncertainty [23].

Since data structures play an important role and greatly influence the computational complexity and efficient implementations of algorithm in mobile robots, [23] indicates that these structures are related to robot representation of the world in the configuration space (C-space) such as the Voronoi diagram, regular grids/occupancy grid, generalized cones, quad-tree and vertex graph. Furthermore, the C-space is usually represented by data structures that show the position and orientations of objects and robots in the workspace area including the free space regions and forbidden regions with obstacles or mazes.

In practice, a route is a finite sequence of straight line courses involving the problem of finding a path with minimal length according to the Euclidean metric [25].Thus, even in autonomous underwater vehicles, the straight line path between the starting position and the goal destination is a good approximation to the optimum path, independently of the eddy structure existing in the area [10].

In search of a data structure that would reduce the number of primitives to be matched, [2] worked with an approach in image segmentation that produced a set of continuous digital lines represented as chain codes which was the common data structure for representing complex segmentation results. Further work would concentrate on path planning to approximate the chains as sequences of digitized straight line segments (DSLS ${ }^{1}$ ), with an algorithm in which the path is made up of straight line segments. Chain code was introduced by Freeman in 1970 [9] as a onepixel-thick boundary descriptor in a grid, and digital straightness was conjectured as well. In this model, given a pixel, the main and immediate neighborhoods of this pixel are shown by symbols, as in Fig. 1.

Fig. 2 shows an example of the string of DSLS in the first quadrant, composed of symbols $a$ and $b$.

\footnotetext{
${ }^{1}$ We use DSLS and other abbreviations, to stand as both the singular and the plural, each one to be grasped from the context.
} 


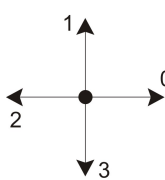

(a)

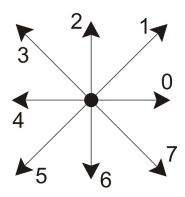

(b)

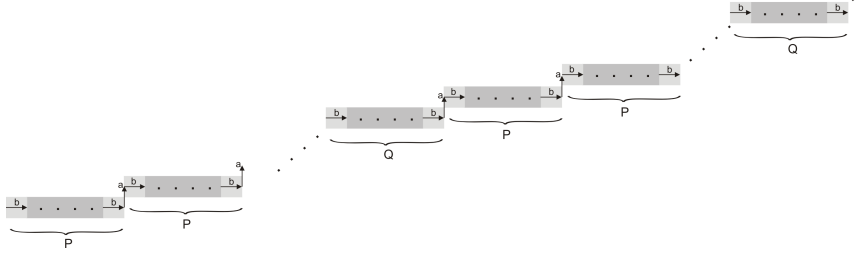

Fig. 2. A generic DSLS in the first quadrant, composed of runs of $P$ and $Q$ symbols $b$, as spaced as possible between codes of $a$, with $P$ and $Q$ constant integers.

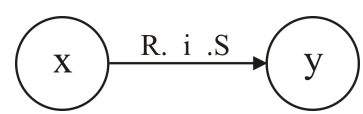

Fig. 3. A generic AFA transition $(x, i): R \rightarrow y: S$, where $R$ and $S$ are optional. of the chain code symbols

the right, the chain code

symbols 0-7 of

neighborhood-8
Understanding the problem from the syntactic point of view involves the concepts of language, grammar and types of grammars ([15]). According to Noam Chomsky, hierarchy dating back to 1956, languages are classified into four different classes: Recursive Languages (or type 0), Context Sensitive Languages (or Type 1), Context-Free Languages (or type 2) and Regular Languages (or Type 3). There are degrees of complexity related to the classes mentioned since class 3 type is a subset of class Type 2 , Type 2 class is a subset of a class type 1 , class and type 1 is a subset of Class 0. Syntactic pattern recognition is one field that uses chain codes together with the rich theoretical background of languages, grammars and automata [30].

However, there are obstacles to employ the theory of languages, grammars and automata in robot navigation because DSLS are actually a very challenging subject incorporating all the dissimilarities and disparities between the discrete and the continuous representations [13].

For instance, DSLS of variable lengths require powerful recursive languages, making it impossible to apply simple formalism for syntactic analysis, such as FSA (finite state automaton) [6] remarks that a regular language is specified by a regular grammar. The concepts of regular language and FSA are equivalent in the sense that for every regular language there is at least one FSA that recognizes it and vice versa [30].

Restricted to trajectories in only four directions (north, south, east or west), [26] investigated state machine applications in robotics introducing a syntactic method that used adaptability to dynamically increase the memory requirements. This work resulted in an architecture based on robot agents implemented by adaptive finite automata (AFA) as motion automata.

Overall, the scrutiny in studies and researches about robot navigation showed that the low representation fidelity using traditional methods based on Cartesian environment maps results in little knowledge stored in the surroundings of the robot [18], commonly achieved by image processing sensors associated with detection of straight lines and arcs.

Yet, among the existing research approaches, syntactic methods are usually considered unsuitable for tasks involving DSLS in robotic navigation. Since [8] and [7] presented capabilities of adaptive techniques related to the formalism of ADSLS in computational geometry, we advance further proposing this formalism applied to robotic navigation utilizing the same AFA, a Turing-powerful device [19], such as motion automaton to direct robots.

This paper is organized as follows. In Section 2, the underlying principles necessary for understanding this study are presented, such as the state-of-the-art of DSLS and codification details. The method involved in this study is described in Sections 3 and 4, regarding automaton implementation and adaptive grids, respectively. Simulations are performed in Section 5 corresponding to proofs of concept aiming at the analysis of this proposal. In Sec- tion 6 , the position of this research within the state of the art is indicated. In Section 7, final considerations are drawn with intended future studies.

\section{FUNDAMENTS}

An adaptive device changes its behavior dynamically in response to input stimuli without interferences from other external agents, including users [19]. Normally, they are made of two layers comprising a non-adaptive underlying mechanism $N D_{0}$, associated to an adaptive layer $A M$, using the same formalism of the first. This growth in complexity profits not only from the notable increment in expressive power of the combination, but also in versatility, as one can choose any consolidated mechanism as the non-adaptive device. An adaptive finite automaton $(A F A)$ is represented by Expression 1 with FSA as $N D_{0}$

$$
A F A=\left(N D_{0}, A M\right) .
$$

The next topic presents a brief review of AFA.

\subsection{Adaptive finite automaton (AFA)}

From Equation 1, AFA is a rule-driven device described by a non-adaptive device, a FSA; associated to an adaptive layer $A M$ comprising adaptive actions that works on the original set of rules $\left(N D_{0}, A M\right)$. $N D_{0}$ characterizes AFA initial configuration. Adaptive actions are calls to parametric adaptive functions (ADF) responsible for self modification procedures. Depending on the stimulus $i$ from input string, linked to an operational step $i$, AFA configuration $N D_{i-1}$ is modified by adaptive actions, resulting that the FSA $N D_{i-1}$ is changed to another FSA $N D_{i}$ belonging to the set $\left\{N D_{0}, N D_{1}, N D_{2}, N D_{3} \ldots N D_{i} \ldots: i \geq 0\right\}$.

Furthermore, the AFA formalism regards elementary adaptive actions to be applied to the transition set of the automaton, so that sets of elementary adaptive actions are abstracted in ADF which interconnects the adaptive layer to $N D_{i}$, as presented in Fig. 3 through generic ADF $R$ and $S$.

Fig. 3 shows the static graphic representation of a generic AFA transition where $x$ is the current state before the transition; $y$ is the state after the transition; $i$ is the input stimulus before the transition; $R$ is an ADF executed before applying the transition; and, finally, $S$ is an ADF executed after applying the transition. Graphically, any ADF $R$ is portrayed by $R \bullet$ in case it is of the before type; likewise, any ADF $S$ is an after type if it happens to be denoted by $\bullet S$.

When it comes to formats, there are three modalities of elementary adaptive actions, specified by a prefix symbol ?, + or -: i) In-

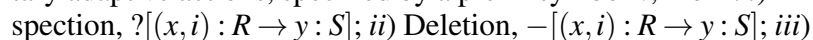
Insertion, $+[(x, i): R \rightarrow y: S]$. Given a certain pattern transition enclosed in brackets, where $[(x, i): R \rightarrow y: S]$ is the pattern to be specified and $R$ and $S$ are optional, the inspection kind searches the current state set for this pattern. The deletion one erases the 
pattern from the current state set; and the insertion kind adds the pattern to the current set of transitions. A provision is made so that the inspection type is executed first, next the deletion, and finally the insertion kind; adding that null transitions have the lowest priority.

About ADF format, in the general case, it has a heading composed of parameters, generators and variables and a body constituted of elementary adaptive actions. All of them are optional; however, if parameters are specified, they have to be supplied to activate the corresponding ADF.

Variables are used in place of any of the components of the elementary adaptive action, further assigned the actual corresponding values in the matching process with the pattern given. Then, after the matching process, variables may be undefined (in case no match is found) or defined (otherwise). Generators are used to assign names to newly created states. Roughly speaking, they are also like special variables, which are automatically assigned unique values as soon as an $\mathrm{ADF}$ is activated. In the activation of an ADF, the assignment of argument values to the parameters occurs, too. Neither generators nor parameters are allowed to change any longer, once assigned.

To differ from variables, generators receive the symbol ${ }^{*}$ as exponent.

\subsection{DSLS background}

A digital arc $S$ is understood as a set of interconnected pixels belonging to a digital image, positioned on a grid such that each point of the set has exactly two neighbors, except two of these points, known as extremes, which have only one neighbor in $S$ [13].

Hence, in neighborhood-4 or neighborhood-8, the chain is a sequence of elements in which each element is a symbol from Fig. 1 that represents the vector joining two neighboring pixels of a digital arch, aiming to represent the digital arch in question.

In his model, Freeman stated that strings representing straight lines must obey three properties in neighborhood-8: (Prop 1) At most two types of symbols, representing directions in the chain code, can be present, and these can differ by unity module eight. (Prop2) For one of these directions, the run length must be 1. One of the two symbols always occurs singly. (Prop3) Successive occurrences of the single symbol are as uniformly spaced as possible among codes of the other value, which occurs in groups. The meaning of Prop1 to Prop 3 is to represent the straight line by a sequence of vectors with multiple slope of $45^{0}$ and the lengths of which are either 1 (when horizontal or vertical), or $\sqrt{2}$ (when diagonal).

As the third property Prop3 was considered somewhat unclear, researches proved that the straightness of a digital arc can be determined by the absence of unevenness in its chain code, necessary and sufficient for meeting the chord property ${ }^{2}$ [12].

The chord property implied establishing a hierarchical structure composed of consecutive numbers corresponding to the runs and runs of runs of the symbols specified by Prop1 and Prop2. This structure of consecutive numbers is expressed by an additional property Prop4. [22] demonstrated that there can be only two possible lengths of these different runs, which are two consecutive integers (for example, $P$ and $P+1$ ).

On the other hand, [13] showed examples of DSLS that violate the regularity implicit in the chord property, commenting that, in practice, Prop3 and Prop4 are inviable in digital arcs. However, it is more reasonable to expect a slight variation in the runs, within a tolerance level, but always keeping the overall slope, thus defining an approximate DSLS. Therefore, a criterion used

\footnotetext{
${ }^{2}$ A digital arc $A$ is said to have the chord property if for every two digital points $c$ and $d$ in $A$, and for each point $p=(x, y)$ on $\overline{c d}$, there is a point $e=(h, k)$ of $A$ such that $\max \{|x-h|,|y-k|\}<1$ where $\overline{c d}$ is the line segment between $c$ and $d$ [22].
}

concentrated on strings that satisfied the first two properties of the conjecture, called monotonic codes, as they represent digital arcs that are either ascending or descending, with reference to coordinate axis $x$ and $y$.

In order to keep the slope of a digital line, the smallest segment of a DSLS is called the Unit of the Straight Line Segment (USLS), resulting in mathematical models [16].

[7] and [6] stated an enhanced method taking into account that the adaptive representation can express changes in the scales of segments. Therefore, an irregular trajectory may be detected as DSLS after it is reviewed in a compatible scale, using metrics. In summary, adaptivity can be an alternative to incorporating the fundamentals of arithmetic discrete geometry to Freeman's model in robot navigation.

\subsection{Codification}

If nothing else is specified, without loss of generality, in this paper neighborhood-4 (seeFig. 1) is the default, so that the symbols of property Propl must be consecutive, module four. More precisely, the symbols that make up strings belong to $\sum=\{a, b, c, d\}$. To satisfy Propl, just consider module 4 along with $a=1, b=0$, $c=3, d=2$, for neighborhood- 4 of Fig. 1 and Fig. 2.

Any string $S=s_{1} \ldots s_{n}, s_{i} \in \sum$ may be represented by its symbol, followed by the indication of its $i$-th element $s_{i}$ giving Expression 2:

$$
S: s_{i} ; i=1,2, \ldots, n .
$$

In Expression 2, $n$ denotes the length of string $S$, which means $|S|=n$. Symbols $s_{i} \in \sum$ may be called tokens, chain code elements or stimuli, too. The null string $n=0$ is represented by $\varepsilon$. If all symbols of $S$ are identical, $s=s_{1}=s_{2}=\ldots=s_{i}=\ldots=s_{n-1}=s_{n}$, a compact representation is $S=s^{n}$. Note that null transitions cause automaton non determinism, then symbols such as $\Delta(\Delta \notin \Sigma)$ may be used in place of $\varepsilon$.

\subsection{Adaptive DSLS (ADSLS)}

AFA implements ADSLS related to digital paths of trajectories to represent the complexities of real world robot scenarios. ADSLS uses a modified chord property for models of higher orders (order $n$ ), thus incorporating tolerances in angle and in length of DSLS. The modified chord property changes neighborhood of chord property into a variable neighborhood function such as $\max \{|x-h|,|y-k|\}<n$, where $n$ is the order of the model that depends on the momentary situation and the length of the segment, to sum up, of the stimuli. That is to say, the neighborhood function of DSLS must have a relatively large width, proportional to the measured length towards the overall linear structure [17].

Regarding techniques for error recovery in this study, it is often convenient to represent the real numbers in a given circumference and not in a straight line, as usual. Especially, from the circumference of unit length, when defining an arbitrary origin point, we represent any point $T$ by its measured distance around the circle in a counterclockwise direction (this by definition). The division of the circle can be from the Farey series in the form of spyrographs described on page 326 of [13].

The techniques of error recovery of syntactic analysis of DSLS employ an approach similar to spyrographs in the form of adaptive loops, such that, by these loops, the circumference is built by states of the AFA, which moves cyclically and continuously through the closed loop. In effect, adaptive loops have their total number of states according to tolerance levels.

In order to simplify the description of automata, take in to account that the abbreviation HTST means a sequence head-to-toe of transitions that consume the same symbol; besides, each state belonging to the sequence may be specified by the first state followed by its respective sequential index. The extremes of a hypothetic DSLS may be truncated or completely out of the global 
structural model. In the former case, the sequence should be accepted; in the latter rejected. Fig. 4 exposes an AFA which tests the first USLS of a DSLS $\left(a^{4} b\right)^{n}$. Parameter $r 4$ is the last state of the HTST starting in $r$. From this arrangement, the AFA removes up to four $\Delta$ transition by ADF RA. Elucidating, each time RA is activated by token $a$, it removes from the automaton one of the $\Delta$ transitions that constitutes the HTST. Furthermore, any token $b$ received conducts the AFA to the final state; conditioned to if more than four tokens $a$ are received, the sequence is rejected. The analysis of the other extreme is quite similar.

2.4.1 Slope errors and length similarity of DSLS. Recognition of DSLS subjected to slope errors is exemplified by $U S L S_{i}=$ $\left\{a^{n} b: 3 \leq n \leq 5\right\}$.

Fig. 4 shows the initial configuration of the automaton prepared to accept truncated $U S L S_{1}$ similar to the last item. With the first token $b$ consumed, ADF $\mathrm{B}$ is activated, which removes transitions of the initial configuration, changing the automaton topology to that of Fig. 5.

Afterwards, the AFA starts to consume the succeeding $U S L S_{i}$ : $i>1$ until the input stream is exhausted. A token $c$ is included just to signalize the end of the DSLS, when the automaton reaches the final state if the process is successful. On the other hand, if more than 5 tokens $a$ are received, $-\left[\left(x_{i}, v r 1\right): v r 2->\right.$ $v r 3: v r 4]$ of RA removes transition of $c$ to the final state, rejecting the sequence.

Strings of Fig. 6 show the performance of the AFA. These strings follow the model $U S L S_{i}=\left\{a^{n} b: 3 \leq n \leq 5\right\}$, truncating $U S L S_{1}$ in some strings, too. Strings out of this model are rejected. Note that the AFA performance does not depend on the length of the input DSLS.

The method to represent and to apply tolerances is by a graph, or a loop such that the number of states of the loop (that is, its size) is changed adaptively in function, for example, of angle $\theta_{S}$ related to axis $x$; besides, $\theta_{S}$ gives the main direction of DSLS $S$, obtaining a syntactic measurement parameter $(1-\psi)$ relative to $S$ [6].

\section{THE METHOD: APPLICATION TO THE AUTOMATA}

Software is modeled in this research as systems of state machines to cover the complete space environment of the robot, scheme adapted from [29]. The group of automata is necessary because there will be several alternative route options varying in angles. Independently of grid resolution changes the group of automata remains fixed either for routes in a relatively limited area or for routes over the complete area as well. Starting from an initial configuration, longer routes will dynamically increase the number of automaton configurations, also increasing the number of automaton states as shown in Fig. 6.

The validation of this study is made by simulations of robot movements associating robotic agents to motion automata, such as AFA, on account of a management system which supplies these agents with data. The technical details by which the management system interconnects itself with AFA and the partitioning criteria to implement AFA are described in the following items.

\subsection{Architecture}

In Expression 1, AFA is represented by two layers: $A F A=\left(N D_{0}, A M\right)$, where $\mathrm{AM}$ is an adaptive layer; an adaptive structure was implemented to simulate navigation scenarios composed of the following layers:

- The System Manager is a hierarchically superior adaptive layer to monitor and to coordinate ADSLS, such as the amount of ADSLS changes according to the stimuli under management of this layer. The System Manager searches information from ADSLS in their respective final states, which indicate if the input string was accepted or rejected;

- The Slave Layer comprises one or more ADSLS and it is commanded by the System Manager or adaptive upper layer.

In the more general case, the upper layer controls the ADSLS of the slave layer by strings of type $W=\Psi C$.

$\Psi$ is a chain code received by the AFA in order that this one adjusts a tolerance band related to the chain $C . \Psi$ corresponds to a factor to compensate for inaccuracies in the digitalization process. The subject of inaccuracies and errors in digitalization is detailed in [8], which lists several papers on this issue.

\subsection{Design patterns}

Topic 2.4.1 illustrated the recognition of DSLS subjected to slope errors, exemplifying by ADSLS of USLS $S_{i}=\left\{a^{n} b: 3 \leq n \leq 5\right\}$ with 3 a transitions and $(5-3) \Delta$ transitions. That adaptive structure (automaton of Fig. 5) is one of the design patterns of this method because to implement other structures from other quadrants, it is just necessary to change the symbols of the pattern conveniently, obtaining an ADSLS with the same topology. Table 1 shows angle ranges of ADSLS and respective expressions of USLS that helps in this procedure.

Table 1. Angle ranges $\theta_{u}$, related to axis $x$.

\begin{tabular}{|c|c|c|}
\hline Sector of ADSLS & Angle Range $(\mathrm{rad})$ & Expression \\
\hline 1 & $0<\theta_{u}<\pi / 4$ & $a b^{n}$ \\
2 & $\pi / 4<\theta_{u}<\pi / 2$ & $b a^{n}$ \\
3 & $\pi / 2<\theta_{u}<3 \pi / 4$ & $d a^{n}$ \\
4 & $3 \pi / 4<\theta_{u}<\pi$ & $a d^{n}$ \\
5 & $\pi<\theta_{u}<5 \pi / 4$ & $c d^{n}$ \\
6 & $5 \pi / 4<\theta_{u}<3 \pi / 2$ & $d c^{n}$ \\
7 & $3 \pi / 2<\theta_{u}<7 \pi / 4$ & $b c^{n}$ \\
8 & $7 \pi / 4<\theta_{u}<2 \pi$ & $c b^{n}$ \\
\hline
\end{tabular}

Simply, for example, to implement an ADSLS for angle range between $5 \pi / 4$ and $3 \pi / 2$ radians, just change the symbols of the pattern of Fig. 5 in the following way: a to $\mathrm{c}$ and $\mathrm{b}$ to $\mathrm{d}$ getting the ADSLS with the same topology (belonging to the third quadrant). In a quadrant, for instance for angles between 0 and $\pi / 2$ radians, grant the validity of properties Prop1 and Prop2, conceiving that symbols change at $\pi / 4$ radians by inspection in Table 1 .

\subsection{Partitioning criteria to implement ADSLS}

The partitioning criteria to implement AFA is in compliance with the angle ranges of each ADSLS to cover $2 \pi$ radians, pondering about angle errors of DSLS and requiring precision, as well. Topic 2.4.1 exemplified the ADSLS with $\operatorname{USLS}_{i}=\left\{a^{n} b: 3 \leq n \leq\right.$ 5\}.

If angles of DSLS are between 0 and $\pi / 2$ radians in this topic, inasmuch as a similar behavior occurs in other quadrants: i) For $0<\theta_{u}<\pi / 4, U S L S_{i}=a b^{n}$, implying $\theta_{u}=\arctan (1 / n)$; ii) For $\pi / 4<\theta_{u}<\pi / 2, U_{S L S}=b a^{n}$, implying $\theta_{u}=\arctan (n)$. Then approximations are by virtue of non-linearity and discontinuity of the function arctangent.

Accordingly, with $n_{\min } \leq n \leq n_{\max }$ for sectors $S 1$, S4, S5, S8, Expression of angle range $\theta_{u}$ of USLS is: $\arctan \left(1 / n_{\max }\right) \leq \theta_{u} \leq$ $\arctan \left(1 / n_{\text {min }}\right)$. With $n_{\text {min }} \leq n \leq n_{\text {max }}$ for sectors $S 2$, S3, S6, S7, Expression of angle range $\theta_{u}$ of USLS is: $\arctan \left(n_{\text {min }}\right) \leq \theta_{u} \leq$ $\arctan \left(n_{\text {max }}\right)$.

\subsection{Implementation of Automata}

In order to maintain a certain angle range, non linearity of arctangent forces the increase of number of states of ADSLS near $(0+k \pi / 2)$ radians, with $k$ integer: $k=0,1,2,3, \ldots$. For angles of sectors 2, 3, 6 and 7, because of discontinuity of arctangent in 


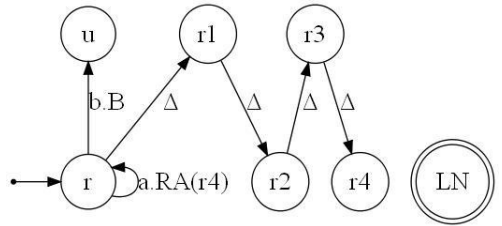

Fig. 4. Initial Configuration for automaton to detect a DSLS considering slope errors, including testing the first USLS of a DSLS from $\left(a^{4} b\right)^{n}$ model

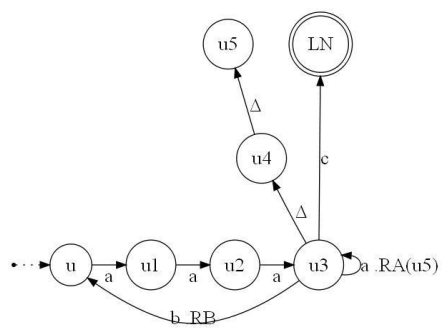

Fig. 5. Configuration of AFA of Fig. 4 after the activation of ADF B

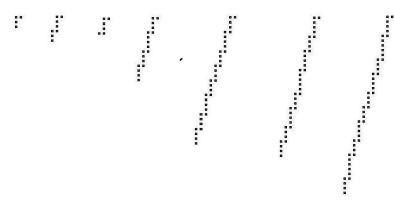

Fig. 6. Examples of DSLS produced by $U S L S=\left\{a^{n} b: 3 \leq n \leq 5\right\}$, accepted by AFA of Fig. 5 $\pm \pi / 2$ radians, angles $\theta_{u}$ of DSLS greater than $\widetilde{\theta}_{1}=\arctan (20)$ are approximated to $+\pi / 2$ or $-\pi / 2$ radians, each one to be analyzed depending on the sector. For angles of sector 1, 4, 5 and 8 , angles $\theta_{u}$ of DSLS smaller than $\widetilde{\theta}_{2}=\arctan (1 / 20)$ are approximated to 0 or $\pi$ radians, to be analyzed depending on the sector. Implemented automata are described below.

3.4.1 ADSLS. For the first quadrant, refering to Table 1, the following ADSLS were selected.

Sector $1\left(a b^{n}\right): 2 \leq n \leq 4$ (Automaton A); $5 \leq n \leq 7$ (Automaton $B$ ); $8 \leq n \leq 12$ (Automaton C); $13 \leq n \leq 20$ (Automaton D).

Sector $2\left(b^{n}\right): 2 \leq n \leq 4$ (Automaton A); $5 \leq n \leq 7$ (Automaton $B$ ); $8 \leq n \leq 12$ (Automaton C); $13 \leq n \leq 20$ (Automaton D).

For the other quadrants, the same topology as that of the ADSLS of the first quadrant was chosen implying each sector has 4 automata: Automaton A, Automaton B, Automaton C, Automaton D

3.4.2 For angles $(0+k \pi / 2)$ radians, with $k=0,1,2,3, \ldots$. For 0 radians, straight line is of the kind: aaaaaaa..., for $\pi / 2$ : bbbbbbb...; for $\pi$ : ddddddd...; for $3 \pi / 2$ : ccccccccc..., requiring 4 automata for the syntactic analysis of these languages. For 0 radians, language of straight line strings is $L=\left\{a^{n}: n>0\right\}$. Fig. 7 shows an automaton which accepts L:

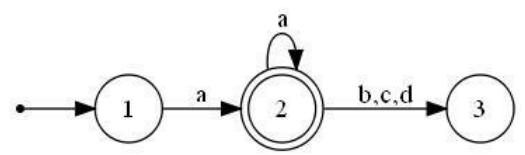

Fig. 7. Finite accepter that recognizes the set of strings belonging to $L=\left\{a^{n}: n>0\right\}$, for 0 radians.

In Fig. 7, with the first symbol a, the automaton goes into state 2 , staying in this state for symbols a, accepting the input string. Any symbol b,c,d which received the automaton enters state 3 , a non-final trap state, signifying the input string is rejected. The other automata have the same topology, changing the symbols conveniently.

3.4.3 For angles $(\pi / 4+k \pi / 2)$ radians, with $k=0,1,2,3, \ldots$. For $\pi / 4$ radians, straight line is of the kind: abababababab....; for $3 \pi / 4$ : $b c b c b c b c b c b c b .$. ; for $5 \pi / 4$ : $d c d c d c d c d c d c d$...; for $7 \pi / 4$ : $c b c b c b c b c b c b c b c b c$.... Fig. 8 is an automaton that accepts strings for $\pi / 4$ radians:

In Fig. 8, with symbols ab or ba, the automaton enters final state 4 or 5 , respectively. In state 4, the input string is accepted as long as symbols ab are received in sequence. Final state 6 allows the automaton to accept an a symbol ending the input string. In state
5, the input string is accepted as long as symbols ba are received in sequence. Final state 7 allows the automaton to accept $a b$ symbol, the last one of the input string. Non-final trap states are not included.

The other automata have the same topology, changing the symbols conveniently.

\section{THE METHOD: APPLICATION TO THE GRID MAP}

Observing that the chain code symbols 0-3 of neighborhood-4 or chain code symbols 0-7 of neighborhood-8 of Fig. 1 are the primitives that define the grid resolution, $[18]$ points out of those grids are considered relatively simple data structures that provide a comprehensive picture of the surfaces in the continuous terrain for robot navigation in a dynamic environment. However, paths formed by grid edges can be longer than true shortest paths in the terrain since their headings are artificially constrained, restricted to multiples of 45 degrees [18].

The improvement in robot navigation is proposed here by the following: i) Bringing ADSLS into play . ii) Concurrently, using adaptive grid resolution. iii) Linked together, applying adaptive changes of orientation angles related to the environment of graphical representation of the chain code symbols 0-3 of neighborhood-4 or chain code symbols 0-7 of neighborhood-8, of Fig. 1.

Item i) above avoids the movement in constrained angles (neighborhood-4: angles multiples of 90 degrees, neighborhood8: angles multiples of 45 degrees). Item ii) varies grid resolution depending on the trajectory, adaptively changing the length of the chain code symbols of Fig. 1. Item iii) is to escape the limitation caused by the discontinuity of the arctangent function, adaptively changing the angle of the chain code symbols of Fig. 1.

Strings resulting from the adaptive scale factor changing the length of primitives are recognized by the same set of automata: spatial variability of grid by adaptivity does not require changing the automata.

By being limited to regular languages, [30] had to introduce a scale normalization factor, which could be the total length of a trajectory pattern. A scale transformation in the work by $[30]$ aims to identify the similarity between contours (trajectories) by their corresponding pattern attributes (descriptors) after undergoing the transformation.

The analysis of situations in robot trajectories depends on the scale involved. If a path $\overline{A B}$ is relatively too short compared with the overall trajectory, it is advantageous to use primitive dimensions large enough to be relatively compatible with the size of segment $\overline{A B}$. If $\overline{A B}$ is relatively large, primitive dimensions have to be short enough to detect shorter segments. Therefore, the adaptive scale proposed by this research reuses, in a new con- 
text, a technique of [30] known since the 1970s granted by improvements in computational power obtained by adaptivity.

\section{EXPERIMENTS}

The minimum grid resolution planned for this work is of the robot dimension order, which involves a question of compatible scale between each step of the robot motion and their respective dimensions.

\subsection{Method proposed by this research}

In the classical method, for moving from a starting point to a destination point, the robot has to find a path for which the computational cost is minimum because this is the path with the shortest distance, too. To compute a minimum cost path, it is necessary to compute robot moves from each cell grid to one of its neighbor cells by changing directions, or transitions, from cell to cell. Afterwards, the algorithm computes the total cost in reaching the destination in one of the shortest paths.

A didactic way to describe the method proposed in this paper is to consider that the robot moves every USLS of the trajectory chain input (segmented in DSLS), instead of moving by each symbol as in the classical method. It implies that the robot travels a path consisting of points corresponding to each USLS. It follows that, given an input with nUSLS: $\left\{U S L S_{1}, U S L S_{2}, U S L S_{3}, U S L S_{4}, U S L S_{5}, \ldots, U S L S_{n}\right\}$, the robot will have $n$ short moves to reach the end point, which means computational cost gains regarding traditional methods.

A better solution, computationally more effective than the earlier one, is driving the robot directly to the final point; therefore, this case involves exchanging information with the robot only about coordinates indicative of the map location only of the end point. In short, the method proposed here uses the ADSLS model since, for moving from a starting point to a destination point, the path is segmented in straight line segments, considerably reducing computational costs. This can be seen in Fig. 9, which shows the same robot in two different positions of the same trajectory, with classical method moves indicated by dashed lines and, in red, the single move by the method proposed by this study.

In all robot figures, the classic method trajectory is indicated in dashed lines and in red is the resulting trajectory proposed by this paper.

\subsection{Adaptive grids}

The effect of adaptive grids may be seen in Fig. 10 that shows the same robot at the end points of their trajectories in two different grids.

Fig. 13 shows a robot in a trajectory segmented into three DSLS (chain 1, 2, 3) in the same scale. Chain 1 is $D S L S_{1}=a^{2} b a^{4} b a^{3} b a^{4} b a^{3} b a^{3} b a^{4} b a^{3} b a^{4} b a^{4} b$ recognized by $A u$ tomaton B-sector2. Chain 2 is DSLS $2=\left(c b^{5}\right)\left(c b^{6}\right)\left(c b^{6}\right)\left(c b^{7}\right)$ recognized by Automaton B-sector8. Chain 3 is DSLS $3=$ $\left(c^{8} b\right)\left(c^{9} b\right)\left(c^{12} b\right)^{4}$ recognized by Automaton C-sector 7 , described in topic 3.4.1 and Table 1

Figure 11 and Fig. 12 is the same robot of Fig. 13, although chain 3 is in scale 0.5 and 1.5 , respectively.

\section{COMPARISON WITH EXISTING TECHNIQUES}

This topic indicates the position of this research within the state of the art, inferred by comparison with selected works as the ones mentioned in item 1 taking in consideration that studies in robot navigation generated two major paradigms for mapping indoor environments: grid-based and topological. An overview of the state of the art is provided by [1].

In the occupancy grid method, space may be referred to the vicinity of the robot described by local parameters, or to the entire space by global parameters normally by exploring the environment [24].

Based on the fact that occupancy grids tends to be both unreliable and computationally expensive, [24] reports experiments with four techniques for position estimation using occupancy grids. [18] highlighted that the trajectories of robots require varying angles of trajectories (labeled as navigation at "anyangle"). Thus, [18] states that the trajectories on the Cartesian grid tend to be not the shortest path (ie, the shortest paths on the terrain) because trajectories are restricted artificially to multiple angles of 45 degrees (8-neighborhood) or multiples of $90 \mathrm{de-}$ grees (4-neighborhood). In another study with the same purpose of angles in variable trajectories, [28] formalizes a search space of alternative routes through a search algorithm on graphs. Problems in world modeling techniques based in grid methods resulted in combining global and local occupancy grids [24] and in topological maps. [27] says that topological maps can be used much more efficiently than grid methods. However, techniques based on topological maps provide a limited accuracy because of the low granularity of the discretization [4]. Since topological maps are considerably difficult to learn in large-scale environments, [27] describes an approach that integrates grid-based and topological approaches.

Hence, an existing tendency is to join local metric maps, especially grid-based, through a global topological map. This tendency is not new, as [14] describes an approach by which metrical information is then incrementally assimilated into local geometric descriptions of places and edges, and finally merged into a global geometric map. With this representation, [14] informs that successful navigation does not depend critically on the choice of sensors and movement actuators, their accuracy, or even on the existence of the geometrical descriptions.

Regarding adaptive techniques using AFA in mapping trajectories, certainly the state of the art is represented by [26] which is restricted to trajectories in only four directions (north, south, east and west), sufficient for its purpose. The motivation of [26] in using adaptivity was due to the high amount of memory for recording information for mapping environments required by path planning systems on grids.

The scheme proposed here also involves the formalization of a search space of routes with certain analogy with [28], but this space is represented in this study by an adaptive neighborhood expressed by AFA states. This study proposes to apply the formalism of ADSLS to enable navigation at any angle as in [18] and [28], however integrating in the framework the positive points obtained by [26] in enabling the use of adaptivity to progressively use system memory, consumed according to the area already mapped.

In addition, the formalism of ADSLS presents other advantages in memory usage, considering that just registering coordinates of only two points, the endpoints of the segments representing digitized trajectories, is enough, and not the record of all the points of the trajectories to map building. These advantages in memory usage can contribute to solving the greatest drawback of grid-based approaches, with their enormous space and time complexity, mentioned by [27].

When it comes to syntactic devices, trajectories varying in angle with relatively any length prevent the use of finite automaton for the purpose of this study and inhibits automaton solutions in data structures for robot navigation. In the opinion of [11], the capability of a more powerful class of formalisms should rejuvenate syntactic research originally pursued in the 70 s-80s.

[20] lists the following trends in devices or algorithms: guided by rules (non-adaptive) whose rule sets are invariable; guided by rules (with basic adaptivity) whose rule sets are variable, and guided by rules (with multi-level hierarchical adaptivity) whose rule sets is variable presenting modifiable adaptive functions. It turns out that this proposal seeks devices and algorithms guided 


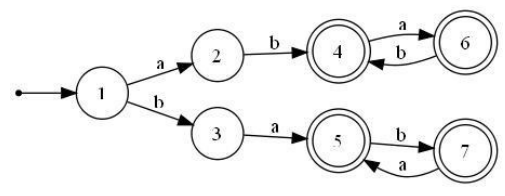

Fig. 8. Finite accepter that recognizes strings for $\pi / 4$ radians.

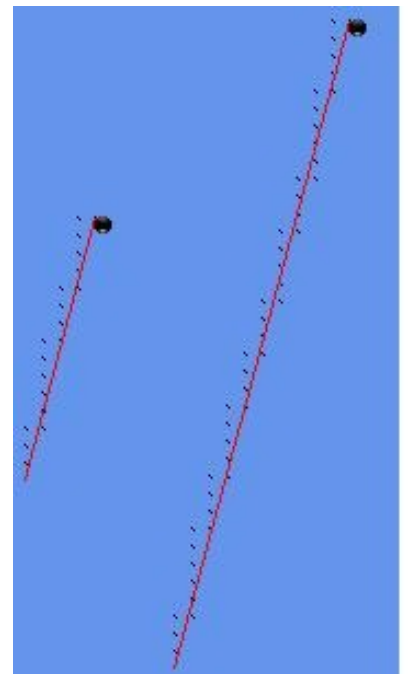

Fig. 9. The figure shows one robot in two different positions of the same trajectory: the classical method trajectory is indicated in dashed lines and in red is the resulting trajectory proposed herein. On the left is an intermediary position, and on the right the end position for the input string $D S L S=$

$a^{3} b a^{5} b a^{3} b a^{4} b a^{3} b a^{3} b a^{4} b a^{3} b a^{5} b a^{4} b$

recognized by the

automaton of Fig.5

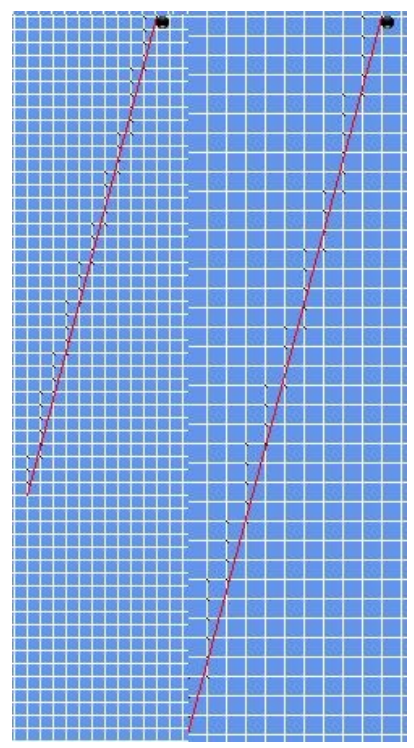

Fig. 10. Figure shows the same robot at the end points of their trajectories in two different grids for input string $D S L S=$ $a^{3} b a^{5} b a^{3} b a^{4} b a^{3} b a^{3} b a^{4} b a^{3} b a^{5} b a^{4} b$ recognized by the automaton of Fig.5 by rules (with multi-level hierarchical adaptivity) whose set of rules is modifiable presenting variable adaptive functions.

Concluding, comparing with the traditional models, without adaptivity, this work has the following objectives: i) Flexibility: In general, traditional methods tend to be computationally complex and too susceptible to errors, spurious interferences, accuracy of sensors and models. Dynamic changes in the vicinity of theoretical models, which alter the functionality of the algorithms depending on the stimuli, allow better characterization of global attributes; ii) Computational Complexity: The introduction of adaptive tolerances simplifies the algorithms compared to methods with invariable behavior, reducing complexity of adaptive algorithms; iii) The advantage of adaptive scale: The adaptive scale allows access to local and global information.

Therefore, this comparison with selected or classical works, represententing tendencies of the state of the art, shows a whole evolutionary potential of this proposal, just by integrating the formalism of adaptive straightness to the traditional models without adaptivity.

\section{FINAL CONSIDERATIONS}

Despite the significance of computational representation, the DSLS being an active area of research for almost half a century as the surveys [13] and [3], this issue has not been explored in robotic navigation for scenarios subjected to spurious influences, with adaptive resources listed in [19]. This research fulfills this gap by incorporating the fundamentals of arithmetic discrete geometry [21] in the syntactic method for robotic navigation, through adaptive techniques.
Indeed, considering variable angles and minute errors of DSLS, to our knowledge, this is the first attempt to introduce AFA in robot navigation. By traditional techniques, automata would have to be implemented a-priori, with high level of complexity to treat errors that cause imprecise models or imprecise scale of DSLS in different angles.

Compared with other methods of digital line representation in navigation, this work showed that the formalism presented in [8] opens new possibilities for robotics navigation, incorporating the main advantages of the ADSLS formalism: i) Simplicity and relative ease of modeling and implementation, associated with high computational power; ii) Models are easy to understand, relatively simple to program and flexible to accept changes in their behavior; iii) Longer trajectories will dynamically increase the storage mapping memory used; iv) Storage memory required to map unknown environments may be reduced drastically by adaptive straight line segmentation of trajectories because just two grid coordinates are necessary to describe a specific digitized straight line path.

Models of DSLS strings were also presented, associated to the corresponding automaton with experiments that demonstrated the simplicity and efficiency of the method, allowing the use of traditional syntactic tooling. The expressive power of the $A D$ SLS formalism incorporates parameters of DSLS such as angle, length and tolerances necessary for navigation algorithms.

A future scope of the idea involves implementations of these data structures in real robots, investigation of navigation algorithms with this proposed schema, for instance by the introduction of 


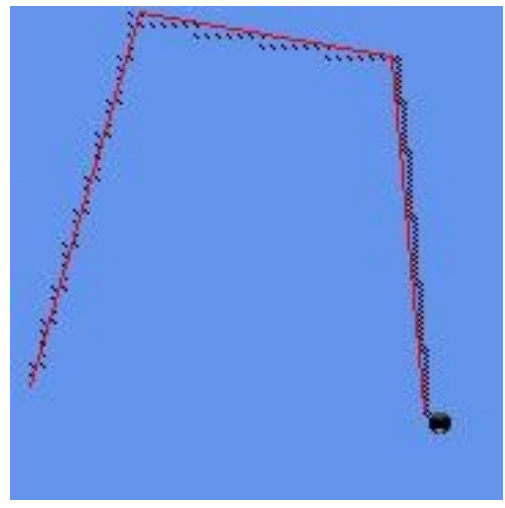

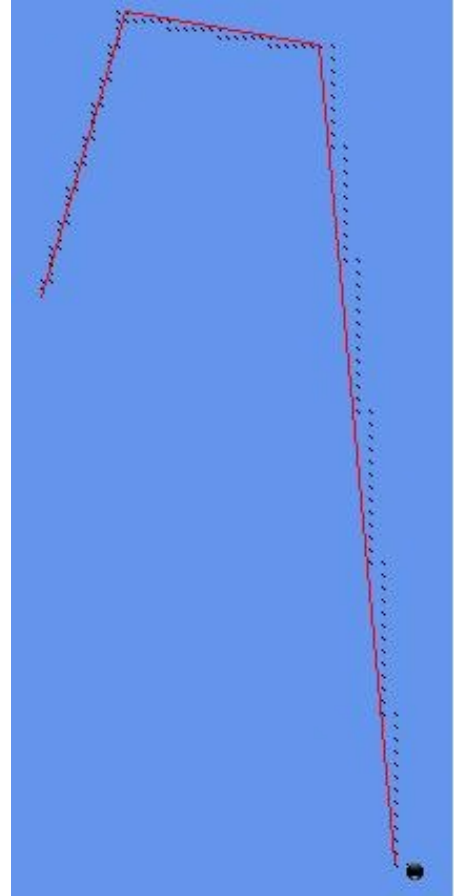

Fig. 12. Figure shows the same robot as in Fig. 13 and

Fig. 11 in a trajectory segmented in three DSLS

(chain 1, 2, 3) with chain 3 in scale 1.5 .

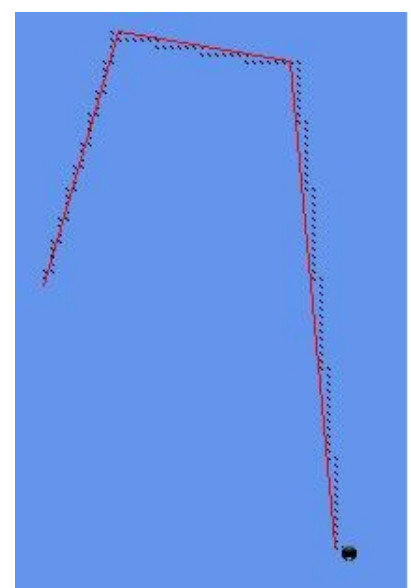

Fig. 13. Figure shows a robot in a trajectory segmented into three DSLS (chain 1, 2, 3) in the same scale, recognized by three automata. obstacles in trajectories, and experimentation of simulated algorithms in real robots.

\section{REFERENCES}

[1] Alejandra Barrera. Advances in Robot Navigation. InTech, 2011.

[2] Rudiger Befit, Dietrich Paulus, and Michael Harbeck. Segmentation of lines and arcs and its application for depth recovery. In Anais..., volume 4, pages 3165 -3168 vol.4. IEEE International Conference on Acoustics, Speech, and Signal Processing, ICASSP-97, april 1997.

[3] Partha Bhowmick and Bhargab B. Bhattacharya. Fast polygonal approximation of digital curves using relaxed straightness properties. IEEE Transactions on Pattern Analysis and Machine Intelligence, 29:1590-1602, September 2007.

[4] Wolfram Burgard, Dieter Fox, Daniel Hennig, and Timo Schmidt. Estimating the absolute position of a mobile robot using position probability grids. In In Proceedings of the Thirteenth National Conference on Artificial Intelligence, Menlo Park, pages 896-901. AAAI Press/MIT Press, 1996.

[5] Yeong-Hwa Chang, Tsu-Tian Lee, and Chang-Huan Liu. On-line approximate cartesian path trajectory planning for robotic manipulators. IEEE Transactions on Systems, Man, and Cybernetics, 22(3), may 1992.

[6] Leoncio C. de Barros Neto, André R. Hirakawa, and Antonio M. A. Massola. Adaptive modeling of digital straightness applied to geometric representation enhancement. International Journal of Computer Applications, 10(2):3139, November 2010. Published by Foundation of Computer Science.
[7] Leoncio C. de Barros Neto, André R. Hirakawa, and Antonio M. A. Massola. An adaptive model applied to digital geometry to enhance segment straightness. IEEE Latin America Transactions, 9:956 - 962, Oct. 2011.

[8] Leoncio Claro de Barros Neto. Modelagem em geometria digital aprimorada por técnicas adaptativas de segmentos de retas. PhD thesis, Escola Politécnica da Universidade de São Paulo (USP), Junho 2011.

[9] H. Freeman. Boundary encoding and processing. Picture Processing and Psychopictorics, pages 241-266, 1970. B.S. Lipkin and A. Rosenfeld, editors, New York, Academic Press, 1970.

[10] Bartolomé Garau, Alberto Alvarez, and Gabriel Oliver. Path planning of autonomous underwater vehicles in current fields with complex spatial variability: an $A^{*}$ approach. In ICRA, pages 194-198. IEEE, 2005.

[11] Feng Han and Song Chun Zhu. Bottom-up/top-down image parsing with attribute grammar. IEEE Transactions on Pattern Analysis and Machine Intelligence, 31(1):59-73, January 2009.

[12] S.H.Y. Hung. On the straightness of digital arcs. IEEE Transactions on Pattern Analysis and Machine Intelligence, PAMI-7(2):203-215, March 1985.

[13] Reinhard Klette and Azriel Rosenfeld. Digital geometry: geometric methods for digital picture analysis. Morgan Kaufmann, 2004.

[14] Benjamin Kuipers and Yung-Tai Byun. A robot exploration and mapping strategy based on a semantic hierarchy of spatial representations. Journal of Robotics and Autonomous Systems, 8:47-63, 1991.

[15] H.R. Lewis and C.H. Papadimitriou. Elements of the theory of computation. Prentice-Hall, 1981. 
[16] Shu Xiang Li and Murray H. Loew. Analysis and modeling of digitized straight-line segments. In Anais..., pages 294-296, Rome, Italy, 1988. Proceedings of International Conference on Pattern Recognition, Publ by IEEE, Piscataway, NJ.

[17] Peter F.M. Nacken. Metric for line segments. IEEE Transactions on Pattern Analysis and Machine Intelligence, 15(12):1312-1318, December 1993.

[18] Alex Nash, Kenny Daniel, Sven Koenig, and Ariel Felner. Theta*: Any-angle path planning on grids. In National Conference on Artificial Intelligence, pages 1177-1183, 2007.

[19] J. J. Neto. Um levantamento da evolução da adaptatividade e da tecnologia adaptativa. Revista IEEE América Latina, 5(7):496-505, Novembro. 2007.

[20] J. J. Neto. Adaptatividade: generalização conceitual. In Anais..., Escola Politécnica da Universidade de São Paulo, 2009. $3^{\circ}$ Workshop de Tecnologia Adaptativa (WTA).

[21] J. P. Reveillès. Géométrie discrète, calcul en nombres entiers et algorithmique. PhD thesis, Université Louis Pasteur, Strasbourg, 1991.

[22] Azriel Rosenfeld. Digital straight line segments. IEEE Transactions on Computers, C-23(12):1264-1269, December 1974.

[23] N. Sariff and N. Buniyamin. An overview of autonomous mobile robot path planning algorithms. In Anais... 4th Student Conference on Research and Development, June 2006.
[24] Bernt Schiele and James L. Crowley. Comparison of position estimation techniques using occupancy grids. In In Proceedings of the 1994 IEEE International Conference on Robotics and Automation, pages 1628-1634, 1994.

[25] J. Sellen. Direction weighted shortest path planning. In Anais..., volume 2, pages 1970-1975 vol.2. IEEE International Conference on Robotics and Automation, may 1995.

[26] M. A. A Sousa, A. R. Hirakawa, and J. J. Neto. Adaptive automata for mapping unknown environments by mobile robots. In Anais... Ibero-American Conference on Artificial Intelligence, Lecture Notes in Artificial Intelligence: Advances in Artificial Intelligence, p.562-571, 2004.

[27] Sebastian Thrun and Arno Bü. Integrating grid-based and topological maps for mobile robot navigation. In Proceedings of the Thirteenth National Conference on Aartificial Intelligence - Volume 2, AAAI'96, pages 944-950. AAAI Press, 1996.

[28] Kamil Tulum, Umut Durak, and Kemal Ider. Situation aware uav mission route planning. In IEEE Aerospace conference, editor, IEEE Aerospace Conference, March 2009.

[29] Ferdinand Wagner, Ruedi Schmuki, Thomas Wagner, and Peter Wolstenhoime. Modeling Software with Finite State Machines: a Practical Approach. Auerbach Publications, 2006.

[30] Kai Ching You and King Sun Fu. A syntactic approach to shape recognition using attributed grammars. IEEE transactions os systems, man, and cybernetics, 9(6):334-345, June 1979. 